\title{
SOME TESTS OF RELATIVISTIC SU(6) SCHEMES*
}

\author{
R. Blankenbecler, M. L. Goldberger, K. Johnson, $†$ and S. B. Treiman \\ Palmer Physical Laboratory, Princeton University, Princeton, New Jersey
}

(Received 8 March 1965)

The problem of generalizing SU(6)-symmetry notions to the relativistic domain has provoked widespread discussion recently, pro and con. ${ }^{1}$ It is now believed, rather generally, that any attempt to directly join the Poincare group to an internal-symmetry group must lead to physical catastrophe, hence that any generalization of SU(6) can at best only represent some sort of approximation to dynamics. Whatever the formal situation may prove to be, for practical purposes one is ultimately interested in symmetry notions, "broken" or otherwise, which are directly applicable to effective $S$-matrix elements at some decent level of approximation. The successes achieved so far for $\mathrm{SU}(6)$ have after all sprung from such direct applications. We are concerned here with such direct tests.

A number of authors $\mathrm{s}^{2-5}$ have recently put forward relativistic generalizations of SU(6) symmetry based on the noncompact group variously called $\mathrm{SU}(12)_{\mathfrak{L}}, \mathrm{U}(12), M(12), \mathrm{SL}(6, c)$, etc. In particular, Bég and $\mathrm{Pais}^{2}$ propose the applicability of their prescriptions to effective vertex functions and $S$-matrix elements. They have recognized the formal difficulties which arise in the framework of local field theory and relativity theory and, therefore, regard their Lorentz completion of $\mathrm{SU}(6)$ as an approximation procedure, hopefully a leading one. At this level, then, one can only ask whether the prescriptions are in reasonable agreement with experiment in various domains of particle physics. One immediate difficulty arises, which transcends the formal details of local field theory. It has been noted by us, and independently by Bég and Pais, ${ }^{6}$ that the relativistic completion of SU(6) comes into conflict with the principle of unitarity of the physical $S$ matrix. This appears to us to be a very serious difficulty. However, the formal demonstration of this conflict gives no measure, quantitatively, of the degree of violation which is involved.

It seems worthwhile, therefore, to set these troubles aside for the moment, and to study the direct implications of the $\mathrm{SU}(12)_{\mathfrak{L}}$ scheme for a variety of experimentally accessible reactions. This must surely be the ultimate test.
Some advance hope is, of course, provided by the successes already achieved for SU(6) itself in quasistatic problems and even in one uncritical, but not unsuccessful, application of SU(6) notions to high-energy forward elastic scattering of mesons on baryons. ${ }^{7}$

In the present note we consider the general class of reactions, for arbitrary scattering angle, $M+B \rightarrow M^{\prime}+B^{\prime}$, where $M$ denotes the pseudoscalar octet, $B$ the baryon octet. In view of the more difficult questions now under discussion, we of course accept for present purposes the validity of the internal symmetry SU(3), and we therefore neglect mass differences among the mesons and among the baryons. For meson-baryon scattering one deals, spatially, with two amplitudes, spin-flip and spin-nonflip. Each, according to SU(3), is a linear combination of seven independent amplitudes. Hence, on $\mathrm{SU}(3)$ the whole complex of meson-baryon scattering reactions is determined by 14 independent complex functions of energy and angle. Concerning the spatial structure, we adopt, in place of the familiar expression for the transition amplitude, an alternative one which is somewhat more convenient for the present purpose; namely,

$$
\bar{u}\left(p_{2}\right)\left[C+i D \gamma_{5} \gamma_{\mu} \epsilon \mu \nu \rho \sigma{ }_{\nu} K_{\rho} \Delta_{\sigma}\right] \mu\left(p_{1}\right),
$$

where $P=\frac{1}{2}\left(P_{1}+P_{2}\right), K=\frac{1}{2}\left(q_{1}+q_{2}\right), \Delta=p_{1}-p_{2}$ $=q_{2}-q_{1}$.

The scalar functions $C$ and $D$ depend on the invariant variables $s=-\left(p_{1}+q_{1}\right)^{2}$ and $t=-\Delta^{2}$. For unpolarized initial baryons, the differential cross section in the center-of-mass system (where $\theta$ is the scattering angle, $k$ the three momentum, $W$ the total energy) is given by

$$
\frac{d \sigma}{d \Omega}=\left(\frac{m}{4 \pi W}\right)^{2}\left(1-t / 4 m^{2}\right)\left[|C|^{2}+\sin ^{2} \theta\left|k^{2} W D\right|^{2}\right]
$$

and the polarization $\langle\vec{\sigma}\rangle$ of the outgoing baryon is given by

$$
\langle\vec{\sigma}\rangle=\overrightarrow{\mathrm{n}} \sin \theta \frac{k^{2} W \operatorname{Im}\left(C D^{*}\right)}{|C|^{2}+\sin ^{2} \theta\left|k^{2} W D\right|^{2}},
$$

when $\vec{n}$ is a unit vector in the direction $\vec{k} \times \vec{k}^{\prime}$. According to the $\mathrm{SU}(12)_{\mathfrak{L}}$ scheme, the bary- 
on octet and decuplet (mass differences being neglected) are collectively described by a totally symmetric tensor $\psi_{A B C}(p)$, where $p$ is the momentum and where each index runs from 1 to 12 . The pseudoscalar-meson octet and vector-meson nonet are collectively described by a mixed tensor $\varphi_{D}{ }^{E}(q)$, where $q$ is the meson momentum. Explicit representations, in terms of the labels of the SU(3) and Lorentz groups, are given in the literature.$^{2-4}$ According to the $\mathrm{SU}(12)_{\mathfrak{L}}$ prescription of Beg and Pais, ${ }^{2}$ the most general structure for meson-baryon scattering is to be gotten by contracting the four tensors involved in all possible ways, each contracted expression being multiplied by an arbitrary function of $s$ and $t$. The general structure is, therefore,

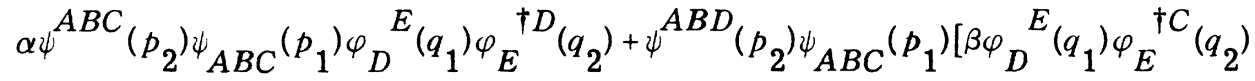

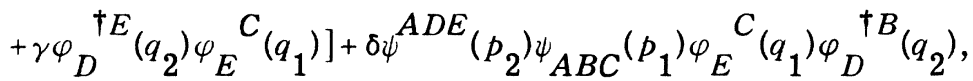

where $\alpha, \beta, \gamma$, and $\delta$ are arbitrary complex functions of $s$ and $t$.

We are concerned here with the part of the above expression which describes scattering of the pseudoscalar-meson octet and baryon octet. The 14 independent amplitudes allowed by $\mathrm{SU}(3)$ collapse here to a total of four independent, complex amplitudes. Evidently, this reduction leads to an enormous number of new predictions for the complex of reactions under discussion.

We shall not write out here the complete structure which emerges when the contractions of Eq. (4) are explicitly carried out. Instead, let us merely note a few of the most striking predictions, some of which can be foreseen just from the general structure of Eq. (4). In particular, we remark that, apart from the arbitrary functions $\alpha, \beta, \gamma, \delta$-which may be complex -the tensor contractions themselves lead to expressions for the two spatial amplitudes $C$ and $D$ of Eq. (1) which are real. Polarization effects, therefore, can arise from interference among the complex functions $\alpha$, $\beta, \gamma, \delta$. For reactions which receive contributions only from a single one of the four contractions, there can then be no polarization on the present scheme. But just from the SU(3) content of the meson and baryon tensors we can easily see in advance that this situation indeed arises for a number of reactions. In any case, on the present scheme one predicts zero polarization for the following reactions [and others related to them through isotopic spin invariance or pure SU(3) invariance]:

$$
\begin{gathered}
K^{-}+p-\bar{K}^{0}+n, \\
K^{+}+n-K^{0}+p,
\end{gathered}
$$

$$
\begin{aligned}
\bar{K}^{0}+n & \rightarrow \Sigma^{+}+\pi^{-}, \\
\pi^{+}+n & \rightarrow \Sigma^{+}+K^{0}, \\
K^{-}+p & \rightarrow \Xi^{-}+K^{+} .
\end{aligned}
$$

Through charge independence the Reactions (7)(9) are, of course, supposed to be, respective$1 y$, identical in detail to the processes

$$
\begin{aligned}
& K^{-}+p \rightarrow \Sigma^{-}+\pi^{+}, \\
& \pi^{-}+p \rightarrow \Sigma^{-}+K^{+}, \\
& \bar{K}^{0}+n \rightarrow \Xi^{0}+K^{0} ;
\end{aligned}
$$

so the predictions concerning the absence of polarization apply for these too. Also, from $\mathrm{SU}(3)$ alone one predicts that (8) and $\left(8^{\prime}\right)$ are equivalent to

$$
K^{-}+p \rightarrow \Xi^{0}+K^{0}
$$

so that again one is led to the expectation of zero polarization here.

A further consequence of the $\mathrm{SU}(12)_{\mathfrak{L}}$ prescription is that the differential cross section for $\left(8^{\prime}\right)$ [hence (8) and (10)] is supposed to be identical with that for $\left(7^{\prime}\right)$ [hence (7)]:

$$
d \sigma\left(K^{-}+p \rightarrow \Sigma^{-}+\pi^{+}\right)=d \sigma\left(\pi^{-}+p \rightarrow \Sigma^{-}+K^{+}\right) .
$$

One also recovers on the present scheme the relations obtained earlier for forward elastic-scattering amplitudes. ${ }^{7}$

Finally, among many other connections implied by the $\mathrm{SU}(12)_{\mathscr{L}}$ prescription, we note the prediction of a definite energy-angle-dependent relation between the differential cross sections for the processes ( $\left.7^{\prime}\right)$ and (9):

$$
\frac{d \sigma\left(K^{-}+p-\Sigma^{-}+\pi^{+}\right)}{d \sigma\left(K^{-}+p \rightarrow \Xi^{-}+K^{+}\right)}=\frac{1}{4}\left[1+\frac{\sin ^{2} \theta k^{4} W^{2}}{R^{2}}\right],
$$


where

$$
\begin{gathered}
12(m+\mu) R=\frac{1}{4}\left[\left(s-m^{2}-\mu^{2}\right)^{2}+\left(s-m^{2}-\mu^{2}+t\right)^{2}\right] \\
-2 m^{2} \mu^{2}+t\left(m^{2}+\mu^{2}+m \mu\right) .
\end{gathered}
$$

For small values of the momentum transfer $t, R$ approaches the value

$$
R \rightarrow m^{2}\left(\omega^{2}-\mu^{2}\right) / 6(m+\mu),
$$

where $\omega$ is the laboratory energy of the incoming meson. We have selected this last example for explicit mention to illustrate a curious feature of the $\mathrm{SU}(12)_{\mathscr{L}}$ scheme, which relates different processes through energy-angle-dependent factors.

For purposes of comparison with experiment, one is faced with the usual problem of dealing with symmetry ideas that are manifestly inexact with regard to mass differences. Indeed, SU(3) symmetry itself does not fare too well in the context of scattering phenomena. ${ }^{8,9}$ If only to avoid those troubles which relate to external mass differences, one ought presumably to effect comparisons with experiment only at high energies, where detailed information is not yet available for all the questions of interest. Nevertheless, the prediction of a null polarization for certain reactions, discussed above, is quite striking, and one may ask whether the experimental indications now available are at all favorable. The answer is decidedly in the negative. In the case of $\mathrm{Re}$ action (9), for example, at $K^{-}$lab momentum $2.3 \mathrm{BeV} / c$ one finds very large $\Xi-$ polariza tions, polarizations which are not far from maximal over a wide range of angles. ${ }^{10} \mathrm{Sim}-$ ilarly, for Reaction (8), at pion lab momentum $1.19 \mathrm{BeV} / c$, there is some evidence for large $\Sigma^{+}$polarization. ${ }^{11}$

These polarization tests of $\mathrm{SU}(12)_{\mathcal{L}}$ have the merit that they are "internal" and do not require the comparison of one reaction with another, where mass differences make ambiguous how the comparison is to be effected when the energies involved are not so high as to make these differences unimportant kinematically [e.g., one would prefer to restrict tests of Eq. (11) to energies in the multi-BeV range]. From the polarization evidence alone, it appears that the $S U(12)_{\mathfrak{L}}$ scheme cannot generally represent a good approximation to particle dynamics. This still leaves open, however, the question why at the same time the scheme incorporates other successful predictions. Bég and Pais have discussed other variations on the theme of boosting $\mathrm{SU}(6)$. It is worth remarking that the affirmative results on forward elastic scattering ${ }^{7}$ are stable under these variations.

\footnotetext{
*Work supported by the U. S. Air Force Office of Research, Air Research and Development Command.

$\dagger$ Permanent address: Massachusetts Institute of Technology, Cambridge, Massachusetts.

${ }^{1} \mathrm{~A}$ nice summary is contained in Sidney Coleman, "Trouble With Relativistic SU(6)" (to be published). It also cites the literature. See also K. Bardakci,
} J. Cornwall, P. G. O. Freund, and B. W. Lee, Phys. Rev. Letters 13, 698 (1964).

${ }^{2}$ M. A. B. Bég and A. Pais, Phys. Rev. Letters 14, 267 (1965); and references therein to earlier papers in which the relativistic completion of $\mathrm{SU}(6)$ is developed.

${ }^{3}$ R. Delbourgo, A. Salam, and J. Strathdee, Proc. Roy. Soc. (London) A284, 146 (1965).

${ }^{4}$ B. Sakita and K. C. Wali, Phys. Rev. Letters 14 , 404 (1965).

${ }^{5}$ K. Bardakci, J. M. Cornwall, P. G. O. Freund, and B. W. Lee, Phys. Rev. Letters 14, 48 (1965).

${ }^{6} \mathrm{M}$. A. B. Bég and A. Pais, to be published.

${ }^{7}$ K. Johnson and S. B. Treiman, Phys. Rev. Letters 14, 189 (1965).

${ }^{8} \mathrm{H}$. Harari and H. J. Lipkin, Phys. Rev. Letters $\underline{13}$, 209 (1964).

${ }^{9}$ S. Meshkov, G. A. Snow, and G. B. Yodh, Phys. Rev. Letters 13,212 (1964).

${ }^{10} \mathrm{An}$ excellent survey has been prepared by M. L. Stevenson, UCRL-11493, 1964 (to be published).

${ }^{11}$ M. M. Nussbaum, R. W. Kraemer, A. Pevsner, M. Block, and A. Kovacs, quoted by F. S. Crawford, in Proceedings of the International Conference on HighEnergy Nuclear Physics, Geneva, 1962, edited by J. Prentki (CERN Scientific Information Service, Geneva, Switzerland, 1962), p. 827. 